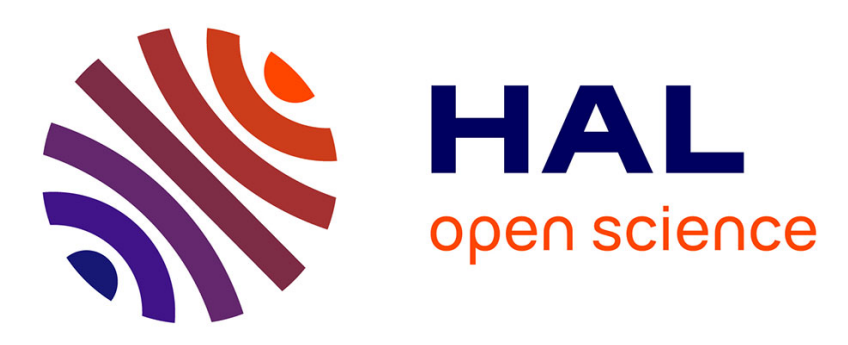

\title{
Onion morphology and microstructure of polyhedral serpentine
}

\author{
Alain Baronnet, Muriel Andréani, O. Grauby, Bertrand Devouard, Serge
}

Nitsche, Damien Chaudanson

\section{To cite this version:}

Alain Baronnet, Muriel Andréani, O. Grauby, Bertrand Devouard, Serge Nitsche, et al.. Onion morphology and microstructure of polyhedral serpentine. 2007. hal-00135139

\section{HAL Id: hal-00135139 \\ https://hal.science/hal-00135139}

Preprint submitted on 6 Mar 2007

HAL is a multi-disciplinary open access archive for the deposit and dissemination of scientific research documents, whether they are published or not. The documents may come from teaching and research institutions in France or abroad, or from public or private research centers.
L'archive ouverte pluridisciplinaire $\mathbf{H A L}$, est destinée au dépôt et à la diffusion de documents scientifiques de niveau recherche, publiés ou non, émanant des établissements d'enseignement et de recherche français ou étrangers, des laboratoires publics ou privés. 


\title{
Onion morphology and microstructure of polyhedral serpentine
}

\author{
Alain Baronnet ${ }^{1, *}$, Muriel Andréani ${ }^{2}$, Olivier Grauby ${ }^{1}$, Bertrand Devouard ${ }^{3}$, Serge Nitsche ${ }^{1}$, \\ and Damien Chaudanson ${ }^{1}$ \\ ${ }^{1}$ Paul Cezanne University and CRMCN-CNRS, Campus Luminy, 13288 Marseille cedex 9, \\ France \\ ${ }^{2 \#}$ Géosciences Marines- IPGP, Place Jussieu, 75252 Paris cedex, France \\ ${ }^{3}$ Laboratoire Magmas et Volcans, Blaise Pascal University - OPGC - CNRS, 5 rue Kessler, - \\ 63000 Clermont-Ferrand, France
}

\begin{abstract}
We describe the shape and internal structure of polyhedral spheroids found in serpentinized peridotites which correspond to a new serpentine microstructure. Serpentine spheroids resemble geodesic domes made of c. 160 to 180 triangular facets. At facet edges, the nested layers bend by c. $14^{\circ}$ along their three $<010>$ crystallographic directions, resulting in an onion-like structure with lateral continuity of the layers. The stacking of the serpentine layers within sectors is controlled by interlayer bonding. These polyhedral onions are made of a novel type of spherical nanostructure for layered materials.
\end{abstract}

Keywords: serpentine minerals, microstructure, onion, spheroid, SEM, TEM.

\footnotetext{
*E-mail: baronnet@crmcn.univ-mrs.fr \# Present address: Laboratoire de Tectonophysique, Montpellier University, Place Eugène Bataillon, 34095 Montpellier Cedex, France.
} 


\section{Introduction}

Serpentine minerals, ideally $\mathrm{Mg}_{3} \mathrm{Si}_{2} \mathrm{O}_{5}(\mathrm{OH})_{4}$, are among a small number of natural layered structures that are known to form aperiodic nanostructures such as cylinders and scrolls (the chrysotile variety; Yada 1967), polygonal tubes (polygonal serpentine; Middleton and Whittaker 1976; Baronnet and Devouard 2005), and cones (Yada and lishi 1977) as well as periodic, flat (the mineral lizardite; Wicks and O'Hanley 1988) and modulated (antigorite; Kunze 1961) crystals. This variety in the wrapping of the building layer of serpentine minerals is strikingly reminiscent of the various nanostructures displayed by carbon (Dresselhaus et al. 2001) and other materials such as $\mathrm{MoS}_{2}$ (Seifert et al. 2000), $\mathrm{WS}_{2}$ (Tenne et al. 1992) or h-BN (Bengu and Marks 2001). The serpentine layer is polar and made of a tetrahedral and an octahedral sheet sharing oxygen atoms. The 'isolated' magnesian octahedral sheet being expected to have a larger in-plane unit-cell than that of the 'isolated' tetrahedral sheet, their assembly to form a serpentine layer may result in a curvature, leaving the tetrahedral sheet inside and the octahedral sheet outside (Pauling 1930). A similar mechanism is invoked to account for the curvature of sulfide misfit layer compounds, which are also known to form a variety of cylindrical, conical or modulated microstructures (Williams and Hide 1988). By comparison to carbon species, an onion-like, spherically wrapped variety of serpentine seemed to be lacking. Serpentine with a globular and polyhedral morphology was previously observed (Mitchell and Putnis 1988, their Fig. 8; Cressey et al. 1993; Zega et al. 2006), and here we expand on those efforts and report details on its morphology and atomic structure, when picked up from terrestrial serpentinites. The serpentine spheroids are studied using highresolution scanning electron microscopy (HRSEM), electron microdiffraction and lattice 
imaging by high-resolution transmission electron microscopy (HRTEM). Our results reveal a novel microstructure in the serpentine group (Baronnet et al., 2006).

Samples and analytical techniques

Polyhedral serpentine presented here comes from optically isotropic veins and altered pyroxenes of two distinct serpentinized peridotite units: i) massive serpentinites exposed in the southern Coast Ranges (Franciscan Complex, California, USA); ii) serpentinites from the inner Piedmont Zone, Western Alps (Italy).

Secondary electron images were recorded at the Centre de Recherche en Matière Condensée et Nanosciences (CRMCN) using a JEOL 6320F scanning electron microscope equipped with a field-emission gun. The insulating serpentine samples were coated with a thin amorphous carbon film for electric conduction, and imaged under $3 \mathrm{kV}$ accelerating voltage for optimum image contrast between the facets of the spheroids. The effective resolution is nearly $10 \mathrm{~nm}$ at that tension and for the C-coating thickness used here.

TEM samples were prepared from dismountable petrographic thin sections. Single-hole TEM copper grids (2x1 mm slot) were glued on the sections for support, detached, and thinned to electron transparency using a GATAN PIPS ion mill. A 20 nm-thick layer of amorphous carbon was finally evaporated onto the sample to provide electric conduction of the specimen surface in the TEM. TEM observations are made through thin wedges of rock that surround holes made by ion milling.

Two transmission electron microscopes at CRMCN were used for the crystallographic and analytical characterization of the serpentine spheroids. A JEOL 2000FX TEM, operated at 200 $\mathrm{kV}(0.28 \mathrm{~nm}$ point-to-point resolution) was used for imaging and selected-area electron 
diffraction (SAED) as well as microanalysis. A Tracor-Northern $5500 \mathrm{X}$-ray energy-dispersive system (EDS) with a Si-Li diode and a ultrathin window collects X-rays at a take-off angle of $72^{\circ}$ from the sample surface. The spatial resolution of microanalyses is c. $30 \mathrm{~nm}$ in the conditions of operations used. Synthetic layer-silicates were used to determine k-factors ( Cliff and Lorimer 1975) for quantifying EDS spectra. A JEOL 3010 TEM at CRMCN, operated at $300 \mathrm{kV}$ (0.21 nm point-to-point resolution) was used to record bright-field, high-resolution images of the serpentine layers. We use rather low magnifications (300 000x) to minimize the electron dose and to delay rapid amorphization of the serpentine.

\section{Results}

We observed serpentine spheroids, $100 \mathrm{~nm}$ to several micrometers in diameter, within pores left by the serpentinization of the orthopyroxene grains in the alpine peridotite (Fig. 1A). In some californian veins and microcracks (Fig. 1B to D), we observed also spheroids acting as filling material commonly intergrown with short nanotubes of chrysotile, less commonly with polygonal serpentine. When their size is greater than one micrometer, these spheroids can be easily recognized from petrographic thin sections as intergrown circular features displaying a black cross when viewed with a petrographic microscope using transmitted light and crossed polars (Fig.1A).

EDS-TEM microanalyses of the Californian sample yielded a structural formula $\left(\mathrm{Mg}_{2.71} \mathrm{Fe}_{0.24} \mathrm{Al}_{0.05}\right)\left(\mathrm{Si}_{1.95} \mathrm{Al}_{0.05}\right) \mathrm{O}_{5}(\mathrm{OH})_{4}$, assuming all iron is in the ferrous state.

Most of the larger spheroids are incomplete, showing only a dome with polygonal facets when developing in a void of the rock (Fig. 1C and D). Imaged with a SEM, they appear as "geodesic domes" (Fig. 1C and D), and sometimes as fully-faceted spheres (inset Fig. 1B). In 
all observed cases, the outer surface is made of an assemblage of nearly equilateral triangular facets. Six such triangular facets meet at each summit of the polyhedron so that a local pseudo-hexagonal rotational symmetry occurs at the corners. We never observed corners displaying five-fold rotational symmetry. We estimate the number of facets for a complete sphere, by dividing the surface area of the sphere by the surface area of a facet, to roughly 160-180. This number appears to be independent of the size of the spheroid, at least for spheroids large enough to be properly observed by SEM (> c. $1 \mu \mathrm{m})$. TEM images and selected area electron-diffraction (SAED) patterns (Fig. 2A) of spheroid tangential sections showed that the triangular facets consist of (001) basal planes of flat serpentine, the edges of the triangles being approximately oriented along the three crystallographically equivalent $<010>$ axes (orthogonal setting). Curved interference fringes frequently occur at the border of many triangular sectors, confirming that triangular pyramidal sectors of serpentine material extend just below each external facet. Cross sections display a concentric, onion-like, nested wrapping of serpentine layers. The common summit of pyramidal sectors is either centered in the complete spheroid or, in the case of incomplete spheroids, such summit lies on a supporting mineral substrate. A remarkable feature is that the layers appear to be mostly continuous all over the periphery of the spheroids. The atomic structure of the serpentine layers can be seen by high-resolution TEM to propagate continuously across contiguous sectors (Fig. 2B). The inward curvature of rounded terminations on all spheroids (Fig. 1C) suggests that the octahedral sheet of the serpentine structure forms the spheroid surface. Sector junctions correspond to a bending of the serpentine structure along the three possible $<010>$ (or b) directions of the silicate, as evidenced by electron diffraction patterns (inset Fig. 2B). The two entangled diffraction patterns are produced by the simultaneous electron diffraction of adjacent sectors selected by 
the SAED aperture. Sector boundaries consist of curved layers similar to those found between sectors of polygonal serpentines (Baronnet and Devouard, 2005). From one sector to the next, layers undergo a tilt of 13 to $14.5^{\circ}$. The angle of bending from one sector to the next appears to be strongly controlled by the crystallographic structure of the serpentine. To ensure proper interlayer bonding and lateral continuity of the layers, there must be a homogeneous shearing of the layer stacking, when going from one sector to the next, and the shearing must be such that successive layers are shifted by a value commensurate with the crystalline structure. In the case of tubular polygonal serpentines, for which bending occurs along the $<100>$ directions at sector boundaries, the intersectorial angle was determined to be 12 or $24^{\circ}$ (Chisholm 1992). In the present case of spheroids, for which bending occurs along $<010>$ directions, the expected value of the angle is $13.4^{\circ}$, inducing an interlayer shift of $+\mathbf{a} / 3$ between successive layers when going from one sector to the next. This value is consistent with the observations from SEM when one measures angles between faces seen on the edge of the spheroids, the tilt angle of (001) lattice planes across sector boundaries (Fig. 2C), and analysis of the electron diffraction patterns (inset Fig. 2B). In contrast, an interlayer shift of -a/3 is not allowed in the serpentine structure (Bailey 1988) because not all H-bonds between successive layers are maintained. This asymmetrical interlayer shift may explain why the facets adopt a triangular shape instead of a hexagonal shape.

Adjacent sectors of the same onion display $1 \mathrm{~T}$ and/or $1 \mathrm{M}$ layer-stacking sequences. When traversing a corner to go from one sector to the one opposite to it (e.g., from sectors labeled 1 to 4 in Fig. 3), the angle appears to be larger. It corresponds to an interlayer shift of $2 \mathbf{a} / 3$, with a predicted intersectorial angle of $27^{\circ}$.

\section{Discussion}


We have described the morphology and structure of polyhedral spheroids of serpentine. Our structural analysis supports the suggested name of "polyhedral serpentine" (Papp 1988, unpublished manuscript; Zega et al. 2006) for this new microstructure of serpentine. Among all reported onion-like structures, natural or synthetic, the nanostructure of polyhedral serpentine is most likely the one that exhibits the best crystallographic control of the morphology. Spherical kaolinite and native or collapsed halloysite, the dioctahedral equivalents of serpentines, are also known to form spheroids with lateral continuity of the layers (Huertas et al. 2004; Sudo 1953; Tomura et al. 1983). However, no crystallographic model has yet been proposed because this mineral dehydrates readily under vacuum. Allophane, another clay mineral, has a spherical structure (Van Olphen 1971) but never develops to form onion-like structures. Although carbon onions have been extensively studied (Iijima 1980; Ugarte 1992; Banhart and Ajayan 1996; Terrones et al. 2002), it is not clear whether there is a crystallographic control of their polygonization (Kroto 1992; Ugarte 1992). Carbon spheroids that develop in cast iron (Minkoff 1983) or carbon balls in some carbonaterich metamorphic rocks (Jaszczak 1994) may have microstructures similar to polyhedral serpentine. However, it remains unclear whether carbon spheroids maintain lateral continuity of the layers, and if so, if a regular polygonization occurs. Moreover, carbon species and related synthetic nanostructures are expected to ensure spherical bending of their layers by the introduction of pentagons within their hexagonal layer, thereby producing spherical nanostructures with icosahedral symmetry. This does not seem to be the case for serpentine, as no corners with five-fold symmetry have been observed. However, it is not geometrically possible to form a perfect spherical structure by connecting equilateral triangles only (Fig. 3). Serpentine spheroids could maintain a spherical morphology while ensuring lateral continuity of the layers by one of the following mechanisms : i) introduction of small-angle tilt 
boundaries between the structures of adjacent facets so as to modify the angles between facets appropriately, ii) slight curvature of the facets and edges of sectors, or a combination of i) and ii).

Like carbon, serpentines are now known to display all types of aperiodic nanostructures (cylindrical or polygonal tubes, cones, and onions) in addition to regular "flat" crystals. Contrary to many other materials, however, the formation of serpentine wrapped structures is possible in conditions closer to the equilibrium in the crystallizing system. As a consequence, wrapped structures of serpentine are often relatively large and thus relatively easy to characterize. The interlayer bonding (hydrogen bonding) of serpentine imposes definite stacking of the layers, which results in strict crystallographic control of the resulting nanostructures. For these reasons, polyhedral serpentine could give extensive insight into the formation and control of carbon multi-walled polyhedral onions and spheroids and of other nested layered structures.

\section{Acknowledgments}

We thank Anne-Marie Boullier (LGIT, Grenoble) for first drawing our attention to this peculiar texture on petrographic thin sections of Californian serpentinites. Marcello Mellini is thanked for having forwarded to us the unpublished manuscript by Papp. The CNRS-INSU is acknowledged for having partly funded the JEOL 3010 transmission electron microscope used for high-resolution imaging and electron diffraction as a National Instrument for Earth Science research projects. Comments of CRMCN members were much helpful to clarify some aspects of the topology of this crystalline object. Marcello Mellini and Thomas Zega provided most helpful reviews. Careful reading of an earlier version by Ellen Siem has been much appreciated. 


\section{References cited}

Bailey, S.W. (1988) Polytypism of 1:1 layer silicates. In : hydrous plyllosilicates, Chap. 2; Ed. S. Bailey; Reviews in Mineralogy, 19, Mineralogical Society of America.

Banhart, F. and Ajayan, P. M. (1996) Carbon onions as nanoscopic pressure cells for diamond formation. Nature, 382, 433-435.

Baronnet, A. and Devouard, B. (2005) Microstructures of common polygonal serpentines from axial HRTEM imaging, electron diffraction, and lattice-simulation data. Canadian Mineralogist, 43, 513-542.

Baronnet, A., Andreani, M., Grauby, O., and Devouard, B. (2006) Polyhedral onions as a novel microstructure of serpentine. Bridging Clays, Programs and abstracts, p. 25.

Bengu, E. and Marks, L.D. (2001) Single-walled BN nanostructures. Physical Review Letters, 86, 2385-2387.

Chisholm, J. E. (1992) The number of sectors in polygonal serpentine. Canadian Mineralogist, 30, 355-365.

Cliff, G. and Lorimer, G.W. (1975) The quantitative analysis of thin specimens. Journal of Microscopy, 103, 203-207.

Cressey, G., Spratt, J. and Cressey, B. (1993) Electron and X-ray petrography of an unusual serpentine from the Tilly Foster mine, Brewster, New York. Canadian Mineralogist, 31, 447458.

Dresselhaus, M.S. and Avouris, Ph. (2001) Introduction to carbon materials research. In Carbon Nanotubes, Dresselhaus, M.S., Dresselhaus, G. and Avouris, Ph. (eds) Topics in Applied Physics, 80, 1-9.

Huertas, F.J., Fiore, S. and Linares, J. (2004) In situ transformation of amorphous gels into spherical aggregates of kaolinite: a HRTEM study. Clays Minerals, 39, 423-431. 
Iijima, S. (1980) Direct observation of the tetrahedral bonding in graphitized carbon black by high-resolution electron microscopy. Journal of Crystal Growth, 50, 675-683.

Jaszczak, J. A. (1994) On the natural occurence of spherical graphite. Rocks and Minerals, 69, 117-118.

Kroto, H.W. (1992) Carbon onions introduces new flavour to fullerene studies. Nature, 359, 670-671.

Kunze, G. (1961) Antigorit: Struckturtheoretische Grundlagen und ihre praktische Bedeutung für die weiters Serpentinforschung. Fortschritte der Mineralogie, 39, 206 -324.

Middleton, A.P. and Whittaker, E.J.W. (1976) The structure of Povlen-type chrysotile.

Canadian Mineralogist, 14, 301-306.

Minkoff, I. (1983) Solidification of spheroidal graphite cast iron. In The Physical Metallurgy of Cast Iron. Chichester, Wiley, 103-133.

Mitchell, R.H. and Putnis, A. (1988) Polygonal serpentine in segregation-textured kimberlite. Canadian Mineralogist, 26, 991-997.

Pauling, L. (1930) The structure of the chlorites. Proceedings of the National Academy of Sciences, USA, 16, 578-582.

Seifert, G., Terrones, H., Terrones, M., Joungnickel, G., Frauenheim, T. (2000) Structure and electronic properties of $\mathrm{MoS}_{2}$ nanotubes. Physical Review Letters, 85, 146-149.

Sudo, T. (1953) Particle shape of a certain clay of hydrated halloysite, as revealed by electron microscope. Mineralogical Journal, 1, 66-68.

Tenne, R., Margulis, L., Genut, M. and Hodes, G. (1992) Polyhedral and cylindrical structures of tungsten disulphide. Nature, 360, 444-446

Terrones, M., Terrones, G. and Terrones, H. (2002) Structure, chirality and formation of giant icosahedral fullerenes and spherical graphitic onions. Structural Chemistry, 13, 373-384

Tomura, S., Shibasaki, Y., Mizuta, H. and Kitamura, M. (1983) Spherical kaolinite: synthesis and mineralogical properties. Clays and Clay Minerals, 31, 413-421. 
Ugarte, D. (1992) Curling and closure of graphitic networks under electron beam irradiation. Nature, 359, 707-709.

Van Olphen, H. (1971) Amorphous clay materials. Science, 171, 90-91.

Wicks, F.J. and O'Hanley, D.S. (1988) Serpentine minerals: structures and petrology. In: Hydrous phyllosilicates (edited by Bailey, S.). Mineralogical Society of America Reviews in Mineralogy, 19, 91-167.

Williams, T.B. and Hide, B.G. (1988) Electron microscopy of cylindrite and franckeite. Physics and Chemistry of Minerals, 15, 521-544.

Yada, K. (1967) Study of chrysotile asbestos by high-resolution electron microscopy. Acta Crystallographica, 23, 704-707.

Yada, K. and Iishi, K. (1977) Growth and microstructures of synthetic chrysotile. American Mineralogist, 62, 958-965.

Zega, T.J., Garvie, L.A.J., Dodony, I., Friedrich, H., Stroud, R.M., and Buseck, P.R. (2006) Polyhedral serpentine grains in CM chondrites. Meteoritics and Planetary Science, 41, 5, 681688.

\section{Figure captions}

Figure 1. Diagnostic aspects of serpentine spheroids. (A) Thin section of a spheroid-like polyhedral serpentine from an altered peridotite, seen under crossed nicols in the petrographic microscope. Each spheroid displays a black cross with arms parallel to the directions of polarization of the two nicols. In such a compact sample, spheroids are intergrown and thus have an irregular outline. (B) SEM image of spheroids of serpentine lining an opened vein which cuts across an altered orthopyroxene grain from a peridotite rock. The void left by alteration allowed spheroids with more regular shapes to form, and triangular facets are visible 
on the larger grains. Inset: an almost completed, tiny spheroid of serpentine, the faceting of which is probably not resolved by SEM. (C and D) Polyhedral morphology of large grains made of triangular facets, roughly equilateral, meeting to form six-fold symmetry corners.

Figure 2. TEM images and SAED patterns of sections made through spheroids of serpentine and its structure modeling. (A) TEM micrograph of a section prepared tangent to a spheroid. Triangular facets are made of flat serpentine layers perpendicular to the view direction and limited by <010> crystallographic orientations (orthogonal setting), as evidenced by SAED (inset) of the black facet. (B and inset) High-resolution TEM imaging and SAED pattern, respectively, of the junction between two adjacent sectors of a large polyhedral serpentine spheroid, observed parallel to the serpentine layers. As for most layer silicates, the $<010>$-type direction of view and bending axis is indicated by the $(0.26 \mathrm{~nm})^{-1}$ distance between diffraction rows in the SAED pattern. Layers propagate continuously at sector boundaries through curvature. The intersectorial angle is c. $14^{\circ}$, as required by the serpentine structure to maintain proper interlayer hydrogen bonding. (C) Perspective sketch of the atomic structure of two successive serpentine layers after bending of $14^{\circ}$ around $<010>$, as expected between any neighboring facet pair. Note the shear of the layer pair (by $+\mathbf{a} / \mathbf{3}$ as calculated from the $14^{\circ}$ bending angle) when crossing the fold hinge, while all H-bonds that connect the layers are maintained in flat parts.

Figure 3. Schematic drawing of the assembly of a few adjacent equilateral triangular sectors. The crystallographic orientation of the serpentine layer is indicated in sector 1. Approximate angles between facet pairs are: $1-2: 14^{\circ} ; 1-3: 24^{\circ} ; 1-4: 27^{\circ}$. This scheme shows that the spherical assembly of equilateral triangles cannot be maintained without modification of 
angles or curvature of the faces and edges. In polyhedral serpentine, it is expected that defects at sector boundaries, and/or slight curvature of the layers allow the polyhedral morphology to be maintained.

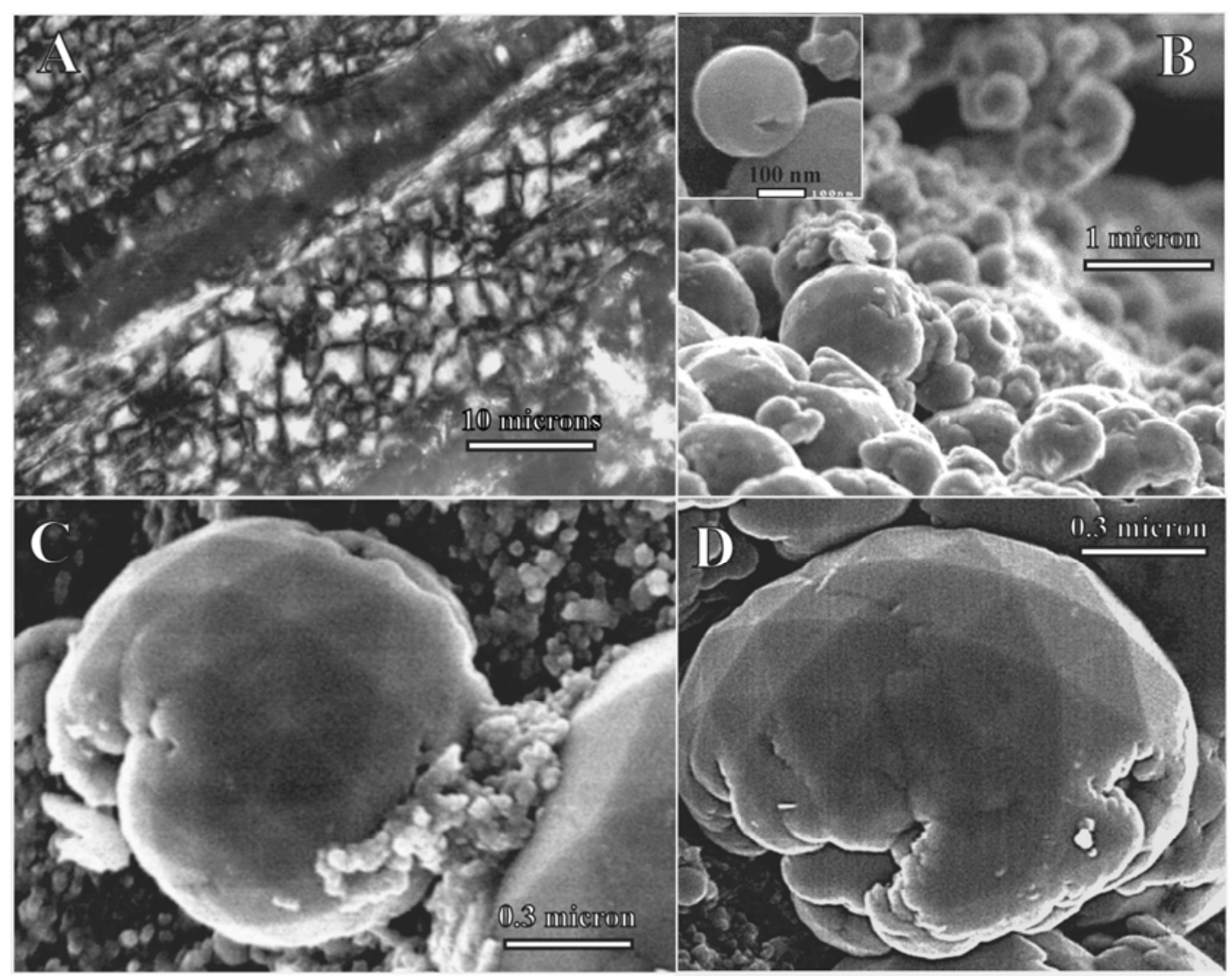

Fig. 1 

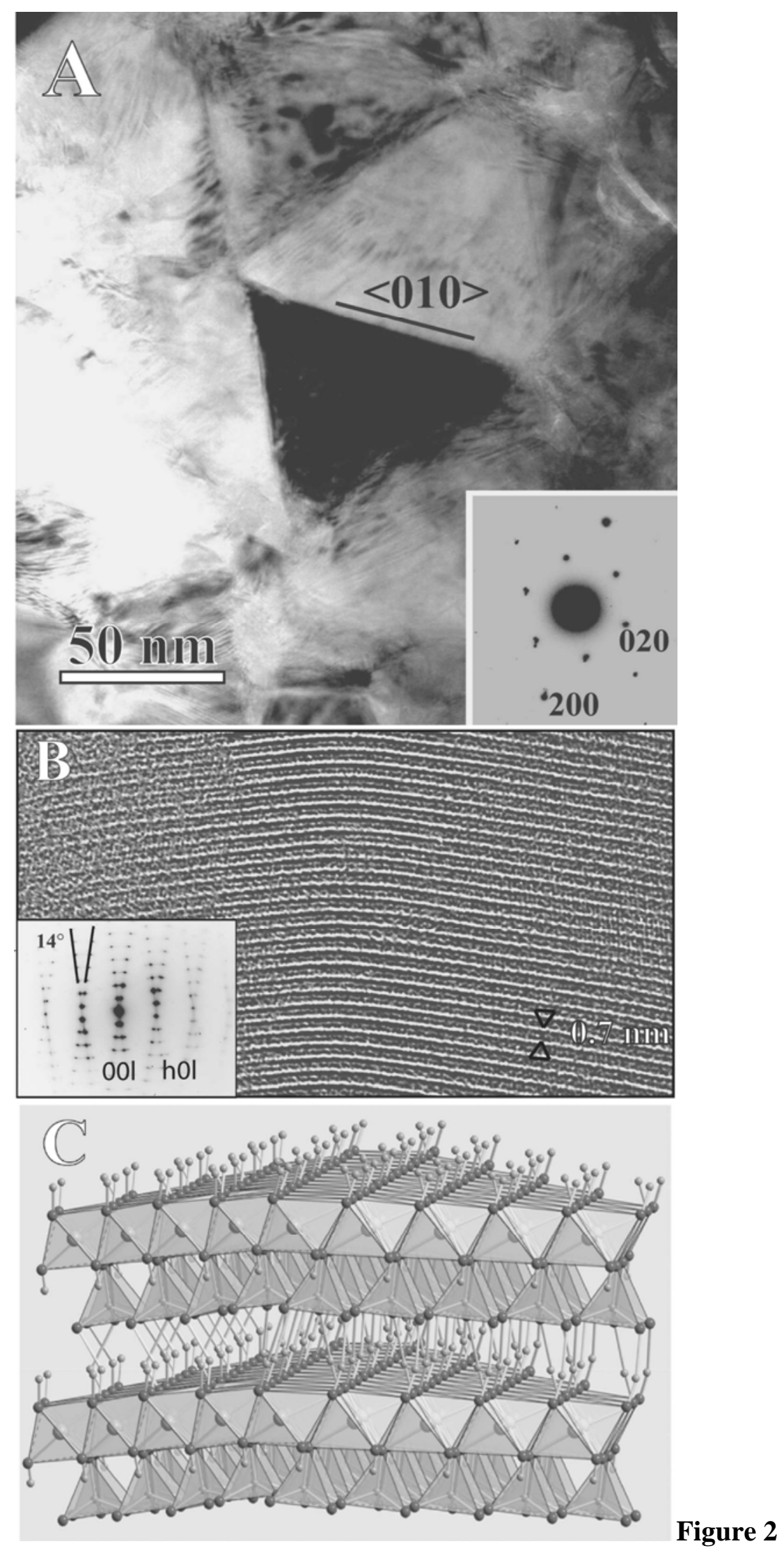


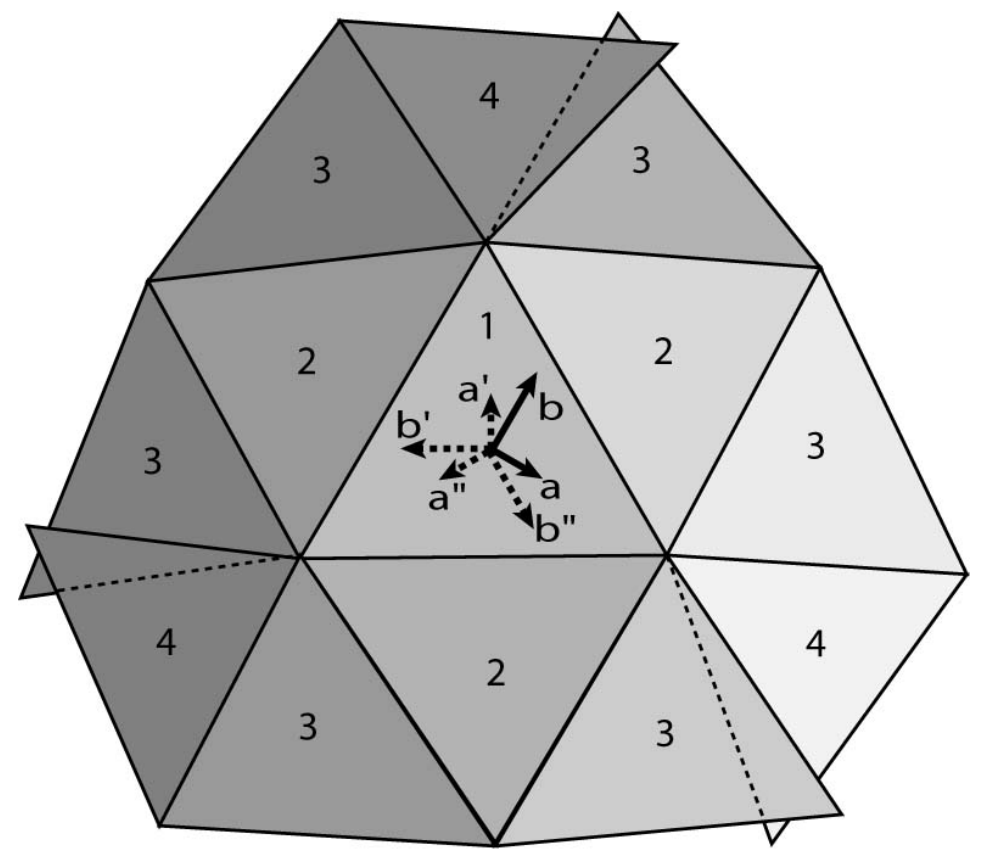

Fig. 3 